\title{
Visualization of Euler deconvolution estimates on traditional and plateau plots
}

Felipe F. Melo* and Valéria C. F. Barbosa, Observatório Nacional

Copyright 2019, SBGf - Sociedade Brasileira de Geofísica

This paper was prepared for presentation during the $16^{\text {th }}$ International Congress of the Brazilian Geophysical Society held in Rio de Janeiro, Brazil, 19-22 August 2019.

Contents of this paper were reviewed by the Technical Committee of the $16^{\text {th }}$ International Congress of the Brazilian Geophysical Society and do not necessarily represent any position of the SBGf, its officers or members. Electronic reproduction or storage of any part of this paper for commercial purposes without the written consent of the Brazilian Geophysical Society is prohibited.

\section{Abstract}

Euler deconvolution is a popular method for interpretation of potential field data. The method estimates the position and the base level for a given nature (i.e., structural index - SI) of a geologic source based on the magnetic dada. These estimates are computed inside a moving data window spanning the whole dataset. The selection of the best estimates is related to the plot of these estimates. Traditionally, for each $\mathrm{SI}$, the estimates of source horizontal coordinates obtained for each position of a moving data window are plotted in the plan view of the study area and the estimates of source depth are represented by different color or size. This traditional plot requires a criterion to select the best estimates and deal with the spread of solutions. In this case, mostly, the baselevel estimates are computed but they are not used or shown. We compare the traditional plot of the estimates from magnetic fields with an alternative plot where, for each SI, every estimate is plotted against the central position of the moving data window. At this plot, when the correct SI is used the solutions form a plateau of constant values. We perform the analysis for synthetic data sets generated by different SI and for a real data set, beyond that; we plot base-level estimates in the traditional way. We run Euler deconvolution over the Diorama anomaly, located in the Goiás Alkaline Province, Brazil, and analyze the estimates over different plots. From the results of both plots we may infer that Diorama anomaly is generated by at least two vertical plugs.

\section{Introduction}

Euler deconvolution (Reid et al., 1990; Barbosa et al., 1999) has become the most popular technique for interpretation of potential-field data because it can process data sets fast. Euler deconvolution works with potentialfield measurements, their gradients, and a given integer number called the structural index (SI), which in turn depends on the nature (type) of the geologic source (Reid et al., 2014; Reid and Thurston, 2014). Mathematically, Euler deconvolution assumes a tentative $\mathrm{SI}$, solves a system of equations in a moving data window scheme and estimates the four parameters: base level, horizontal and vertical positions of a geologic source.

Traditionally, for each tentative SI, one map is generated where the estimates of the horizontal and vertical coordinates obtained by Euler deconvolution are plotted.
Specifically, the Euler deconvolution estimates of the horizontal coordinates are plotted in the plan view $(x-y$ plane) of the study area (Reid et al., 1990), and usually vertical estimates are plotted in different color or size. In this case, base-level estimates are usually neglected. When the correct SI is used the estimates cluster over the source (Thompson, 1982). The cumbersome with this plot is the large amount of solutions (Barbosa and Silva, 2011). In order to deal with this spray of solutions and define the correct SI some authors developed some criteria, e.g., Thompson (1982) and Reid et al. (1990). FitzGerald et al. (2004) provided an overview on best practices to select the solutions of Euler deconvolution.

On the other hand, to define the correct SI, Barbosa et al. (1999) used the minimum correlation between base-level estimates and the observed anomaly. Alternatively, to the traditional plot, Silva and Barbosa (2003) proposed to plot each coordinate estimate against the central position of the moving data window. When the correct SI is assumed, depth estimates define a plateau over the source; however horizontal estimates always define a plateau over the source (Silva et al., 2001) regardless the assumed SI. Melo et al. (2013) took advantage of this behavior to locate automatically the horizontal plateaus. Melo and Barbosa (2018) worked with base-level estimates on this plateau plot and showed that over the source the depth and base-level estimates define plateaus if the correct SI is assumed, and no interfering anomalies are present. In case of interfering anomalies, the minimum standard deviation of base-level estimates over a plateau defines the correct SI. The plateau is delineated either by the depth or horizontal estimates.

In this work, we analyze Euler deconvolution solutions displayed in the traditional (Reid et al., 1990) and in an alternative way, the plateau plot (Silva and Barbosa, 2003; Melo and Barbosa, 2018). Furthermore, we analyze baselevel estimates plotted in the traditional way. To this end, we generate magnetic anomalies from single-point sources related to different SI. We simulate two scenarios: a single source and two sources with distinct SI. Application on real data from part of the Goiás Alkaline Province, Brazil, shows that both plots lead to similar results and we infer that at least two vertical plugs generate the Diorama anomaly.

\section{Method}

Reid et al. (1990) defined the Euler deconvolution as

$\left(x-x_{o}\right) \frac{\partial h}{\partial x}+\left(y-y_{o}\right) \frac{\partial h}{\partial y}+\left(z-z_{o}\right) \frac{\partial h}{\partial z}=\eta(b-h)$,

where $\eta$ is the SI, $b$ is a base level or background value, $x_{o}, y_{o}$ and $z_{o}$ are the source positions, $x, y$ and $z$ are the observation positions, $h=h(x, y, z)$ is the total-field anomaly and $\partial h / \partial x, \partial h / \partial y$ and $\partial h / \partial z$ are the gradients with respect to the variables $x, y$ and $z$, respectively. 
Solving equation 1 , we use a tentative SI, in a moving datawindow scheme, and the estimated parameters are $\hat{x}_{o}$ and $\hat{y}_{o}$ (horizontal positions of the source), $\hat{z}_{o}$ (vertical position of the source) and $\hat{b}$ (base level). Each position of the moving data window produces the four estimates and these estimates are used to define the source.

Figure 1a shows the traditional plot of the estimates for one SI and some positions of the moving data window such as displayed in Reid et al. (1990), the kth position is highlighted. The estimates $\hat{x}_{o}, \hat{y}_{o}$ are plotted at its plan $(x$, $y$ ) position, $\hat{z}_{o}$ is plotted using color or size. Usually the estimates $\hat{b}$ are neglected, although they are calculated in equation 1. In this work, we deal with the spray of solutions using the methodology of Uieda et al. $(2013 ; 2014)$ to select the best solutions. These authors, implemented a filter that keeps only a percentage of the solutions, ranked by the average uncertainty of the estimate (Beiki and Pedersen, 2010). Figure $1 \mathrm{~b}$ shows the plateau plot of the estimates for one SI and the kth position of the moving data window such as displayed in Silva and Barbosa (2003) and Melo and Barbosa (2018). Each estimate $\hat{x}_{o}, \hat{y}_{o}, \hat{z}_{o}$ and $\hat{b}$ is plotted against the central position $(x, y)$ of the moving data window producing four maps. Hence, this plot uses all estimates; it does not discard or filter any estimate. Summarizing, for one assumed $\mathrm{SI}$ the traditional plot (Figure 1a) produces one map of filtered $\hat{x}_{o}, \hat{y}_{o}$ and $\hat{z}_{o}$ estimates ( $\hat{b}$ are neglected), while the plateau plot (Figure 1b) produces four maps of $\hat{x}_{o}, \hat{y}_{o}, \hat{z}_{o}$ and $\hat{b}$ estimates. a)

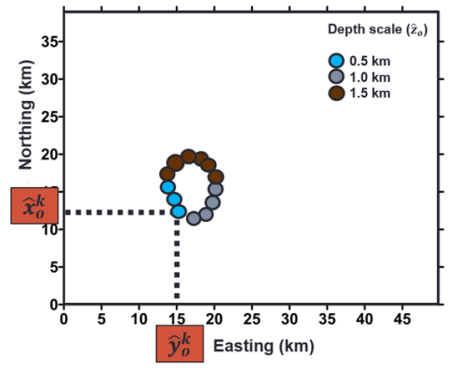

b)
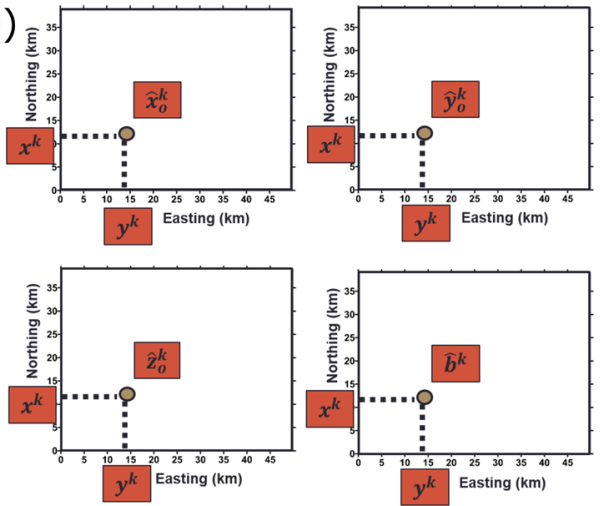

Figure 1 - Plot of Euler deconvolution estimates for one structural index. a) Traditional plot of some positions of the moving data window, the kth position is highlighted. The estimates $\hat{x}_{o}, \hat{y}_{o}$ and $\hat{z}_{o}$ are plotted at its plan $(x, y)$ position and $\hat{b}$ is neglected. b) Plateau plot of one position of the moving data window. Each estimate $\hat{x}_{o}, \hat{y}_{o}, \hat{z}_{o}$ and $\hat{b}$ is plotted against the central position $(x, y)$ of the moving data window.

\section{Synthetic test - single source}

Figure 2a shows a sketch of a survey where the source is a prism with equal horizontal dimensions of $0.1 \mathrm{~km}$, top at $0.4 \mathrm{~km}$ and base at $100 \mathrm{~km}$. This source simulates a singlepoint source that resembles a pole-like source, which has the $\mathrm{SI}=2$. The prism is magnetized with intensity of $8 \mathrm{~A} / \mathrm{m}$ and the simulated main geomagnetic field (main field) is vertical. We simulated a survey for a grid of $65 \times 60$ points equally spaced at each $0.1 \mathrm{~km}$ and the flight height is 0.1 $\mathrm{km}$. Figure $2 \mathrm{~b}$ shows the simulated total-field anomaly, the red square outlines the source.
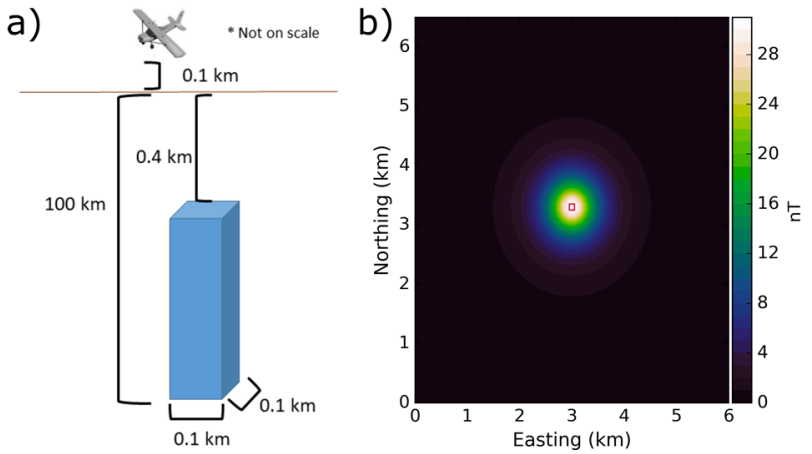

Figure 2 - Sketch of a survey on a profile over the source and the simulated magnetic anomaly. a) Prism with equal horizontal dimensions of $0.1 \mathrm{~km}$, top at $0.4 \mathrm{~km}$ and base at $100 \mathrm{~km}$, the flight height is $0.1 \mathrm{~km}$. b) Total-field anomaly generated by (a), the red square outlines the source.

We run Euler deconvolution with a moving data window of $9 \times 9$ points solving equation 1 for each position of the moving data window. We stress that we used the same results for both plots, but the visualization of the results are distinct. For the traditional plot, we kept the best $5 \%$ of estimates, or 195 estimates. While for the plateau plot, we use all estimates.

Figure 3 shows the traditional plot where the estimates (shown in color) are displayed over the map of the magnetic anomaly (Figure $2 \mathrm{~b}$ ) shown in grayscale. Figure $3 a$ and $3 b$ shows the depth the base-level estimates, assuming $\mathrm{SI}=2$. Notice that in both cases the estimates cluster over the source (outlined in red square) because the $\mathrm{SI}=2$ is the correct one for the simulated source and it is an isolated anomaly, therefore both estimates point to the correct SI. Moreover, depth estimates are correct and define the top of the source, as expected. By assuming a wrong $\mathrm{SI}=3$, Figure $3 \mathrm{c}$ and $3 \mathrm{~d}$ shows the depth and the base-level estimates. Unlike the case presuming the correct SI $=2$, both estimates spread over the source because the $\mathrm{SI}=3$ is not the correct for this source. Moreover, depth estimates are overestimated in this case.

Figure 4 shows the plateau plot of the Euler solutions. Figure $4 \mathrm{a}$ and $4 \mathrm{~b}$ shows $x$ - and $y$-estimates, respectively. Over the source, the plateaus of horizontal estimates are characteristic of this plot, assuming any tentative SI. Figure $4 \mathrm{c}$ shows depth estimates assuming $\mathrm{SI}=2$. Notice the plateau of solutions over the source; these estimates retrieve the true depth $(0.4 \mathrm{~km})$ of the simulated prism. Figure $4 d$ shows base-level estimates, assuming the correct $\mathrm{SI}=2$, these estimates all form a plateau and have a small standard deviation compared to estimates using 
other SI when computed over the plateau of depth estimates (Figure 4c). By assuming a wrong $\mathrm{SI}=3$, the depth (Figure 4e) and the base-level (Figure 4f) estimates do not form a plateau. If the tentative $\mathrm{SI}$ is greater than the correct one (as shown by Figure $4 \mathrm{e}$ and $4 \mathrm{f}$ ) the depth and base-level estimates form protuberances. On the other hand, if the tentative SI is smaller than the correct one (not shown) the depth and base-level estimates form concavities.
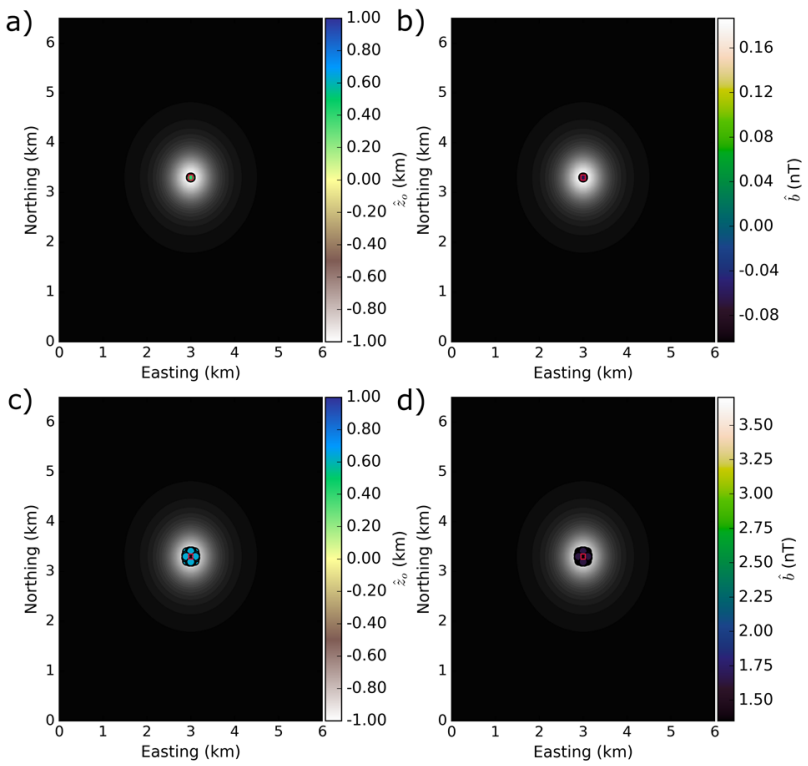

Figure 3 - Traditional plot of Euler deconvolution estimates, over the anomaly in grayscale, The red square outlines the source. a) Depth and b) base-level estimates assuming $\mathrm{SI}=2$, the correct one for this source. c) Depth and d) base-level estimates assuming a wrong $S I=3$.

\section{Synthetic test - sources with distinct SI}

Figure 5a shows a sketch of a survey on a profile over two sources. The southernmost source is a prism with equal dimensions of $0.5 \mathrm{~km}$, centered at $1.4 \mathrm{~km}$. The northernmost prism has equal horizontal dimensions of 0.3 $\mathrm{km}$, top at $0.6 \mathrm{~km}$ and base at $100 \mathrm{~km}$. These sources simulates single-point sources that resembles a dipole-like source, which has the $\mathrm{SI}=3$, and a pole-like source, which has the $\mathrm{SI}=2$, respectively. The compact prism is magnetized with intensity of $10 \mathrm{~A} / \mathrm{m}$ and the elongated prism is magnetized with intensity of $3 \mathrm{~A} / \mathrm{m}$. The sources are assumed to have induced magnetization with inclination of $60^{\circ}$ and declination of $0^{\circ}$. We simulated a survey for a grid of $120 \times 100$ points equally spaced at each $0.2 \mathrm{~km}$ and the survey height is at $z=0 \mathrm{~km}$. The anomaly was corrupted with pseudorandom Gaussian noise with zero mean and standard deviation of $0.25 \mathrm{nT}$. Figure $5 \mathrm{~b}$ shows the simulated total-feld anomaly, the red squares outline the sources. We run Euler deconvolution with a moving data window of $5 \times 5$ points.
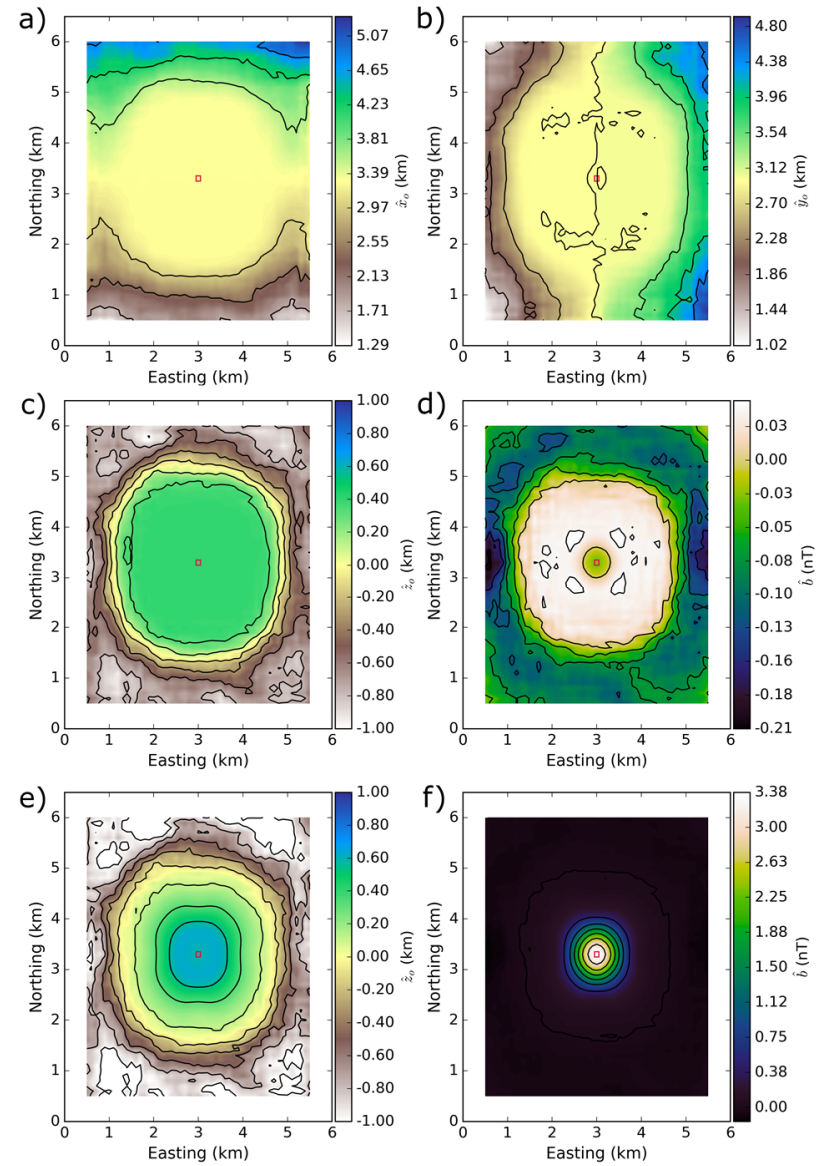

Figure 4 - Plateau plot of Euler deconvolution estimates. a) $x$ - and b) $y$-estimates. c) Depth and d) base-level estimates assuming the correct $S I=2$. The correct $S I$ generates constant estimates over the sources forming plateaus. e) Depth and f) base-level estimates assuming a wrong $S I=3$. The wrong SI does not generate plateaus.
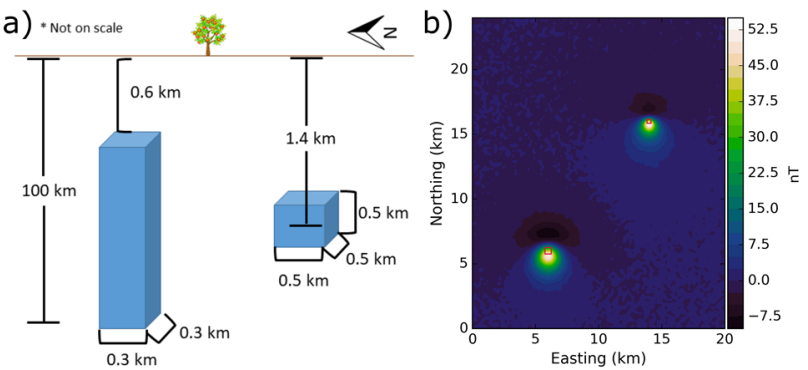

Figure 5 - Sketch of a survey on a profile over the sources and the simulated magnetic anomaly. a) On the left, a prism with equal horizontal dimensions of $0.3 \mathrm{~km}$, top at 0.6 $\mathrm{km}$ and base at $100 \mathrm{~km}$. On the right, a prism with equal dimensions of $0.5 \mathrm{~km}$, centered at $1.4 \mathrm{~km}$. The survey is at $z=0 \mathrm{~km}$. b) Noise-corrupted total-field anomaly generated by the sources shown in a). The red squares outline the sources. 
a)

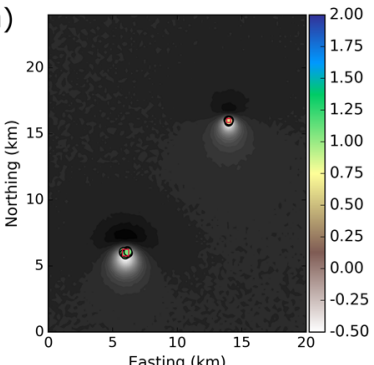

c)

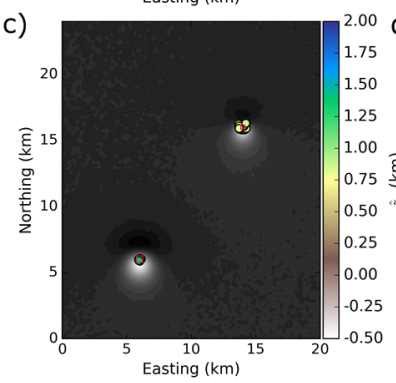

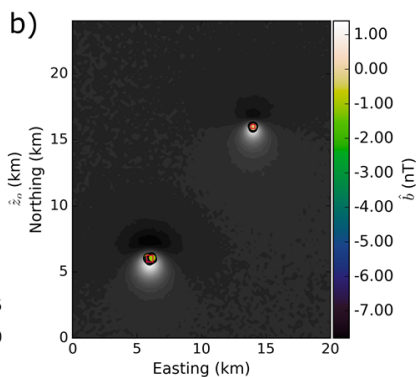

d)

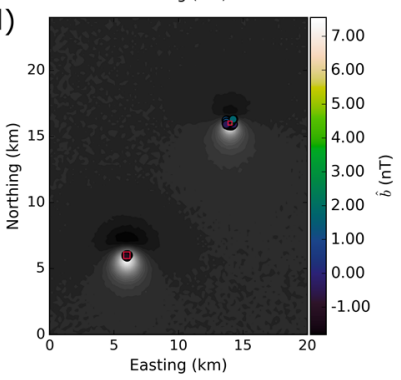

Figure 6 - Traditional plot of Euler deconvolution estimates, over the anomaly in grayscale, and the red squares outline the sources. a) Depth and b) base-level estimates assuming $S I=2$, the correct one for the northernmost source. c) Depth and d) base-level estimates assuming $S I=3$, the correct one for the southernmost source. Assuming the correct SI, the estimates cluster over the source.

For the plateau plot, Figure $7 \mathrm{a}$ and $7 \mathrm{~b}$ shows $x$ - and $y$ estimates, respectively, assuming $\mathrm{SI}=2$. Over the sources, the plateaus of horizontal estimates define the positions of the sources, assuming any tentative SI. Figure $7 \mathrm{c}$ shows depth estimates assuming $\mathrm{SI}=2$. Notice the plateau of solutions over the northernmost anomaly; these estimates have the correct values and define the correct SI. The depth estimates over the southernmost anomaly form a protuberance and underestimate the estimated depth. Figure 7d shows base-level estimates, assuming the correct $\mathrm{SI}=2$, over the northernmost anomaly these estimates indicate the correct $\mathrm{SI}$, form a plateau and have a small standard deviation compared to estimates using other SI when computed over the plateau of depth estimates (Figure 7c). Figure 7e shows depth estimates assuming $\mathrm{SI}=3$, the plateau of solutions is now over the southernmost source defining the correct SI and depth position. Over the northernmost anomaly, the depth estimates form a concavity and the values are overestimated. Figure $7 f$ shows base-level estimates assuming $\mathrm{SI}=3$, the plateau of solutions is over the southernmost anomaly while the estimates over the northernmost source form a concavity. As pointed before, the minimum standard deviation of base-level estimates over the depth plateaus define the correct SI.

\section{Application to real data set}

The Goiás Alkaline Province (GAP) is a region in the central part of Brazil subject to mafic-alkaline magmatism (Marangoni and Mantovani, 2013). The study area is in the northern part of the GAP (Melo et al., 2013; Melo and Barbosa, 2018), this region is characterized by maficultramafic alkaline complexes; these plutonic intrusions

present remanent magnetization (Dutra et al., 2014; Marangoni et al., 2016).
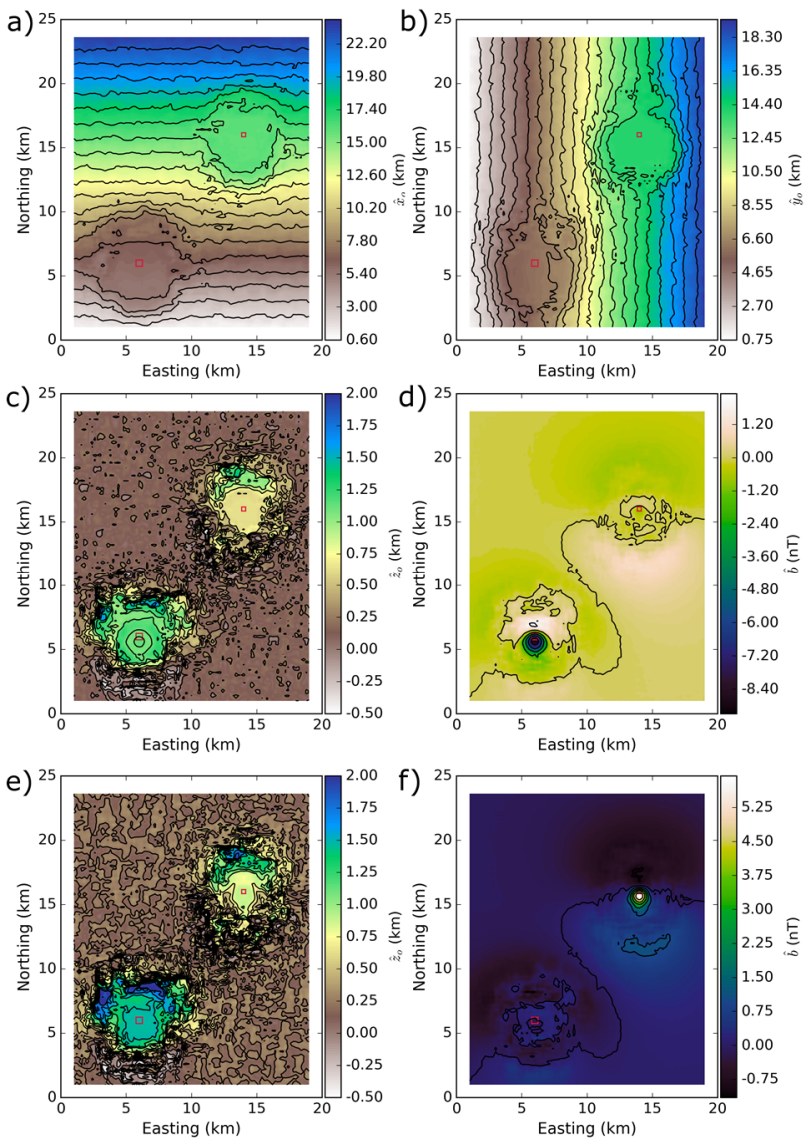

Figure 7 - Plateau plot of Euler deconvolution estimates. a) $x$ - and b) $y$-estimates. c) Depth and d) base-level estimates assuming the $S I=2$, the correct one for the northernmost source. e) Depth and f) base-level estimates assuming $S I=3$, the correct one for the southernmost source.

The real aeromagnetic data (yellow area in Figure 8a) were acquired in 2004 (CPRM, 2004) over Goiás state. The flight lines in the north-south direction were acquired every $500 \mathrm{~m}$, the tie lines were acquired every $5 \mathrm{~km}$ and the flight height was approximately constant at $-100 \mathrm{~m}$. The data set has 105 points northing and 97 points easting is gridded with the same size in the $x$ - and $y$-directions, $125 \mathrm{~m}$.

We focus our study in the Diorama anomaly (blue dot in Figure 8a); in this area are common subvolcanic intrusions, dikes, plugs, and sills of picrite (Dutra et al., 2012). Figure $8 \mathrm{~b}$ shows the total-field anomaly of Diorama anomaly. The shape of the anomaly (Figure $8 b$ ) suggests two sources produced by strong and a weak dipoles, respectively, at the central and northern portions of the anomaly

We run Euler deconvolution with a moving data window size of $9 \times 9$ grid points. For the traditional plot, Figure $8 \mathrm{c}$ and $8 \mathrm{~d}$ shows depth and base-level estimates assuming SI $=2$, and Figure $8 \mathrm{e}$ and $8 \mathrm{f}$ shows depth and base-level estimates assuming $\mathrm{SI}=3$. We kept $7 \%$ of the solutions, or 712 estimates, and at least two sources are identifiable by the clustering of the solutions. In this case, keeping 
fewer solutions vanishes the estimates plotted over the northernmost anomaly. Either via depth or base-level estimates, both results indicate that the correct SI for the sources is $\mathrm{SI}=2$. However, a detailed investigation with fewer solutions, maybe clipping the data, is necessary to make sure of the interpretation. For the plateau plot, Figure $8 \mathrm{~g}$ and $8 \mathrm{~h}$ shows depth and base-level estimates assuming $\mathrm{SI}=2$, and Figure $8 \mathrm{i}$ and $8 \mathrm{j}$ shows depth and base-level estimates assuming $\mathrm{SI}=3$. In depth estimates, we identified three plateaus over the anomaly Diorama and pointed them with white arrows in Figure $8 \mathrm{~g}$. These are the same results obtained by Melo and Barbosa (2018), these authors computed the $\mathrm{SI}=2$ as the correct for these sources, on these plateaus, based on the minimum standard deviation of base level-estimates.

\section{Conclusions}

We compared the traditional and the plateau plots of Euler deconvolution estimates. This is an attempt to clarify the plateau plots and a step in order to understand base-level estimates in the traditional plot. In the synthetic tests with isolated sources, we have shown that both depth and base-level estimates can be used to define the correct SI in the traditional or plateau plot. We applied the criterion to select the best solutions in depth estimates, and from the selected estimates, the corresponding base-level estimates were plotted. However, this criterion can be used directly on base-level estimates or a new criterion can be defined directly from base-level estimates. A next step of this study also includes tests in interfering anomalies to define the limitations of these plots and take advantage of the best on them. Application of Euler deconvolution to a real data set, the Diorama anomaly, and visualization in both plots indicate that, at least, more than one vertical plug generates the anomaly.

\section{Acknowledgments}

F.F. Melo was supported by a Phd scholarship from FAPERJ (grant E-26/200.532/2018). V.C.F. Barbosa was supported by fellowships from: CNPQ (grant 307135/20144) and FAPERJ (grant E-26/203.091/2016). F.F. Melo thanks Rodrigo S. Canário for continuous motivation on the topic. The authors thank CPRM for permission to use the aeromagnetic data set.

\section{References}

Barbosa, V. C. F., J. B. C. Silva, and W. E. Medeiros, 1999, Stability analysis and improvement of structural index estimation in Euler deconvolution: Geophysics, 64, 48-60.

Barbosa, V. C. F., and J. B. C. Silva, 2011, Reconstruction of geologic bodies in depth associated with a sedimentary basin using gravity and magnetic data: Geophysical Prospecting, 59, 1021-1034.

Beiki, M., and L. B. Pedersen (2010), Eigenvector analysis of gravity gradient tensor to locate geologic bodies: Geophysics, 75, 6, I37.

CPRM, 2004, Project: Aerogeophysical survey of Goiás state, first phase: Survey and data processing: Final report, Rio de Janeiro, SGM/MME/CPRM and SIC/SGM/FUNMINERAL/Goiás State, Brazil, vol. 22 and maps (in Portuguese).
Dutra, A. C., Y. R. Marangoni, and T. C. Junqueira-Brod, 2012, Investigation of the Goiás Alkaline Province, Central Brazil: Application of gravity and magnetic methods: Journal of South American Earth Sciences, 33, 43-55.

Dutra, A. C., Y. R. Marangoni, and R. I. F. Trindade, 2014, Aeromagnetic and physical-chemical properties of some complexes from Goiás Alkaline Province: Brazilian Journal of Geology, 44, 361-373.

FitzGerald, D., A. B. Reid, and P. Mclnerney, 2004, New discrimination techniques for Euler deconvolution: Computers \& Geosciences, 30, 461-469.

Marangoni, Y. R., and M. S. M. Mantovani, 2013, Geophysical signatures of the alkaline intrusions bordering the Paraná Basin: Journal of South American Earth Sciences, 41, 83-98.

Marangoni, Y. R., H. Zhang, and H. J. Ferreira, 2016, Gravity and magnetic integrated data interpretation of the Córrego dos Bois complex, Goiás Alkaline Province, central Brazil: Revista Brasileira de Geofísica, 33, 599610.

Melo, F. F., V. C. F. Barbosa, L. Uieda, V. C. Oliveira, Jr., and J. B. C. Silva, 2013, Estimating the nature and the horizontal and vertical positions of $3 \mathrm{D}$ magnetic sources using Euler deconvolution: Geophysics, 78, no. 6, J87J98.

Melo, F. F., and V. C. Barbosa, 2018, Correct structural index in Euler deconvolution via base-level estimates: Geophysics, 83, 6, J87-J98.

Reid, A. B., J. M. Allsop, H. Granser, A. J. Millett, and I. W. Somerton, 1990, Magnetic interpretation in three dimensions using Euler deconvolution: Geophysics, 55, 80-91.

Reid, A. B., J. Ebbing, and S. J. Webb, 2014, Avoidable Euler errors - The use and abuse of Euler deconvolution applied to potential fields: Geophysical Prospecting, 62, 1162-1168.

Reid, A. B., and J. B. Thurston, 2014, The structural index in gravity and magnetic interpretation: Errors, uses, and abuses: Geophysics, 79, 4, J61-J66.

Silva, J. B. C., V. C. F. Barbosa, and W. E. Medeiros, 2001, Scattering, symmetry, and bias analysis of source-position estimates in Euler deconvolution and its practical implications: Geophysics, 66, 1149-1156.

Silva, J. B. C., and V. C. F. Barbosa, 2003, 3D Euler deconvolution: Theoretical basis for automatically selecting good solutions: Geophysics, 68, 1962-1968.

Thompson, D. T., 1982, EULDPH: A new technique for making computer-assisted depth estimates from magnetic data: Geophysics, 47, 31-37.

Uieda, L., V. C. Oilveira Jr., and V. C. F. Barbosa, 2013, Modeling the Earth with fatiando a terra. In: Proceedings of the 12th Python in Science Conference (SciPy 2013), pp. 96-103.

Uieda, L., V. C. Oliveira, Jr., and V. C. F. Barbosa, 2014, Geophysical tutorial: Euler deconvolution of potential-field data: The Leading Edge, 33, 448-450. 
a)
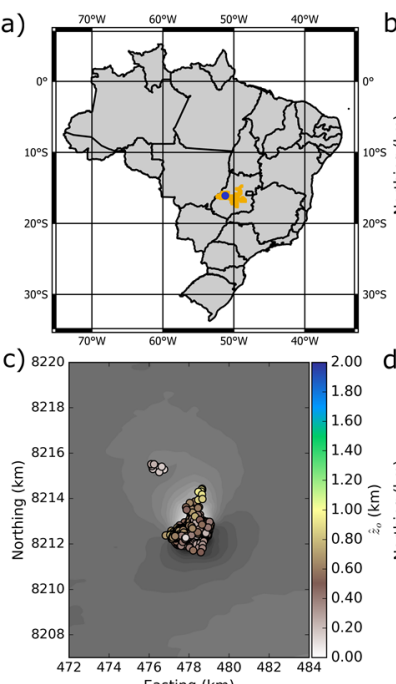

Easting $(\mathrm{km})$
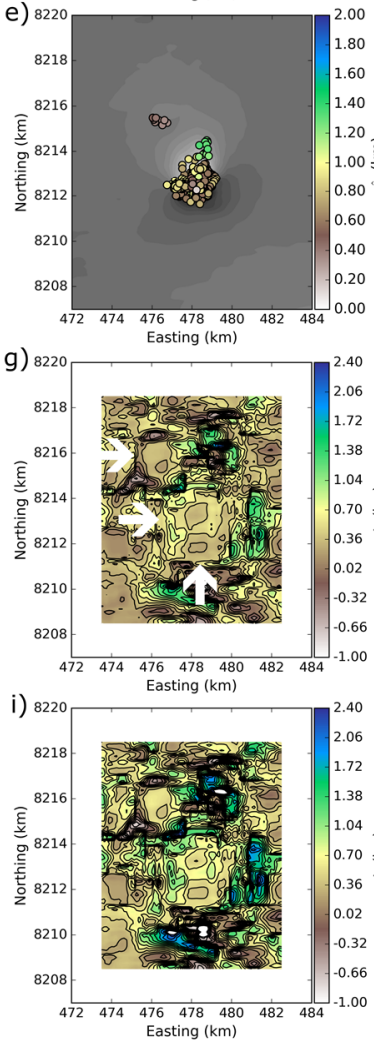
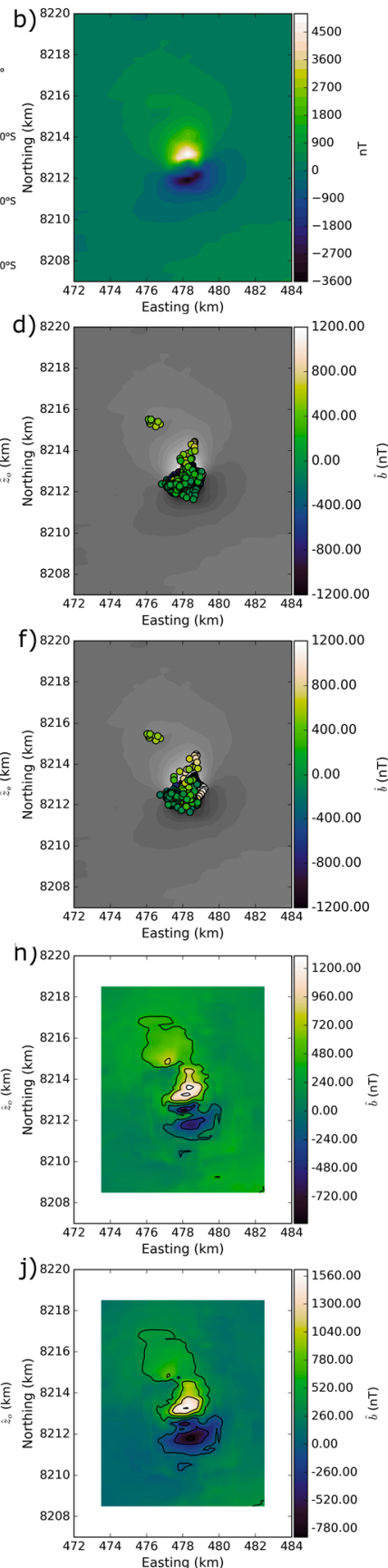

Figure 8 - Real data application - Diorama anomaly and depth estimates of Euler deconvolution. a) Survey in yellow and study area in blue dot. b) Diorama anomaly. Traditional plot assuming $\mathrm{SI}=2$ for c) depth and d) base-level estimates. Traditional plot assuming $\mathrm{SI}=3$ for $\mathrm{e}$ ) depth and f) base-level estimates. Plateau plot for $g$ ) depth and $h$ ) base-level estimates assuming $\mathrm{SI}=2$, and i) depth and $j$ ) base level estimates assuming $S I=3$. White arrows indicate three plateaus over the anomaly. 